\title{
Choroidal Melanoma in a Dog
}

\author{
Yasutsugu MIWA ${ }^{1)}$, Satoru MATSUNAGA ${ }^{1)}$, Kumiko KATO'), Hiroyuki OGAWA ${ }^{1)}$, Hiroyuki NAKAYAMA ${ }^{2)}$, \\ Saori TSUJIMOTO ${ }^{3)}$ and Nobuo SASAKI ${ }^{4)}$
}

${ }^{1)}$ Laboratories of Veterinary Emergency Medicine, ${ }^{2)}$ Veterinary Pathology, ${ }^{4)}$ Veterinary Surgery, Graduate School of Agricultural and Life Sciences, The University of Tokyo, 1-1-1 Yayoi, Bunkyo-ku, Tokyo 113-8657 and ${ }^{3)}$ Yoshioka Animal Hospital, 1059-2 Onsui, Astugi-shi, Kanagawa 243-0033, Japan

(Received 5 January 2005/Accepted 14 April 2005)

ABSTRACT. A 7-year-old intact female golden Retriever was referred for evaluation of an intraorbital mass of the left eye. Based on ophthalmoscopy, ultrasonography and magnetic resonance imaging (MRI), the tentative diagnosis was made as an intraocular neoplasia, especially choroidal melanoma. The orbital exenteration of the affected eye was performed. The mass was histologically diagnosed as malignant choroidal melanoma. No signs of recurrence and metastasis were detected by thoracic radiographs, blood examinations and MR images, and the dog was clinically healthy for 23 months after operation.

KEY WORDS: canine, choroidal melanoma, magnetic resonance imaging (MRI).

Melanomas are the most common primary ocular tumor in dogs, although primary ocular tumors are relatively rare [1]. Most of primary melanomas in dogs arise from the anterior uveal tract such as the iris and ciliary body $[1,4$, $11]$, and primary choroidal melanoma is less common among primary ocular melanomas [2,9]. Previous studies documented $4 \%$ of canine ocular melanomas to be of the choroidal origin and the behavioral and cytologic features were benign [3, 11]. Another report on benign choroidal melanoma in a dog showed that the tumor lesion did not change for 7 years, similar to that of human uveal nevus [10]. But the other case of canine choroidal melanoma with metastasis was reported [4]. The present paper describes ophthalmoscopy, magnetic resonance imaging (MRI), histopathology and a 23-month follow up of a choroidal melanoma in a dog.

A 7-year-old intact female golden Retriever was referred to Veterinary Medical Center (VMC) at the University of Tokyo for evaluation of an intraorbital mass of the left eye. Two months prior to referral, the owner noticed the blindness of the left eye of the dog and admitted to a practitioner. Opthalmic examinations revealed mydriasis and loss of menace reflex and direct pupilary light reflex in the left eye. Consensual pupilary light reflex was lost in the right eye. Then the patient was referred to another practitioner to examine the cause of the vision loss of the left eye, where the black pigmented and edematous retina around the optic nerve disk were found on ophthalmoscopy and diagnosed as inflammation of the nerve disk. The dog was treated with the taper dose of prednisone with an initial dose of $2 \mathrm{mg} / \mathrm{kg} /$ day. Two weeks later, the dog received ophthalmoscopy and was revealed that the edematous area was reduced but the black-pigmented area was enlarged and rose up toward

\footnotetext{
* Correspondence to: SASAKi, N., Laboratory of Veterinary Surgery, Graduate school of Agricultural and Life Sciences, The University of Tokyo, 1-1-1 Yayoi, Bunkyo-ku, Tokyo 1138657 Japan.
}

the vitreous. Ultrasonogram of the affected eye revealed the solid mass lesion which overlaying the optic disk area. Then the dog was referred to VMC for the detail evaluation of the solid mass by MRI.

On physical examination, the dog was clinically normal. Complete blood count and blood chemistry revealed no abnormal findings. On ophthalmic examination, the menace reflex and the pupilary light reflex were lost as seen in the practitioners. The intraocular pressure was within the normal range in both eyes; $12 \mathrm{mmHg}$ in the right eye and 13 $\mathrm{mmHg}$ in the left eye, respectively. Ophthalmoscopy revealed the increased size of the pigmented area over the optic disk (Fig. 1). No evidence of the retinal detachment was present. MR images were obtained with a 0.3-Tesla

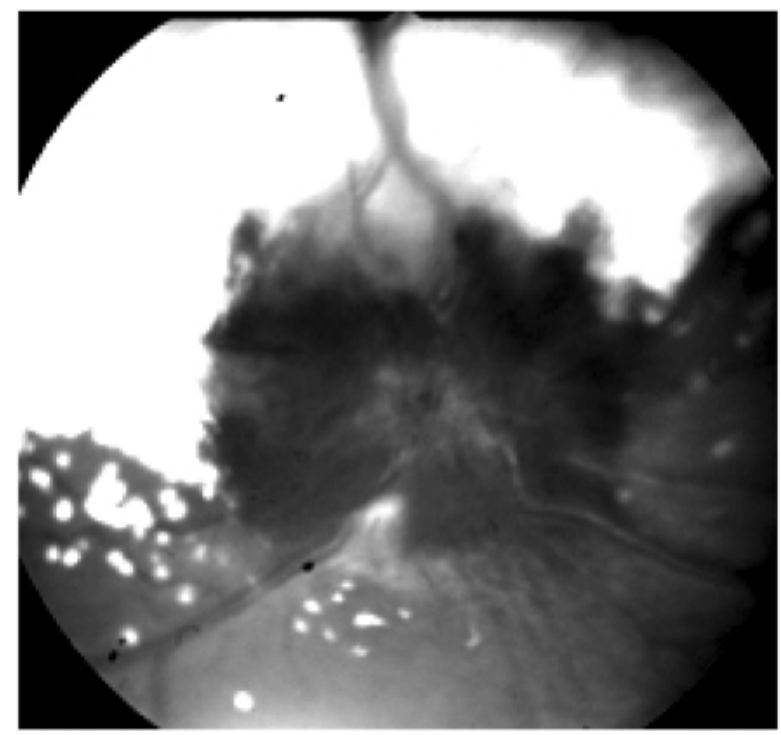

Fig. 1. Funduscopic examination demonstrating the black-pigmented area around the optic disc in the left eye. 

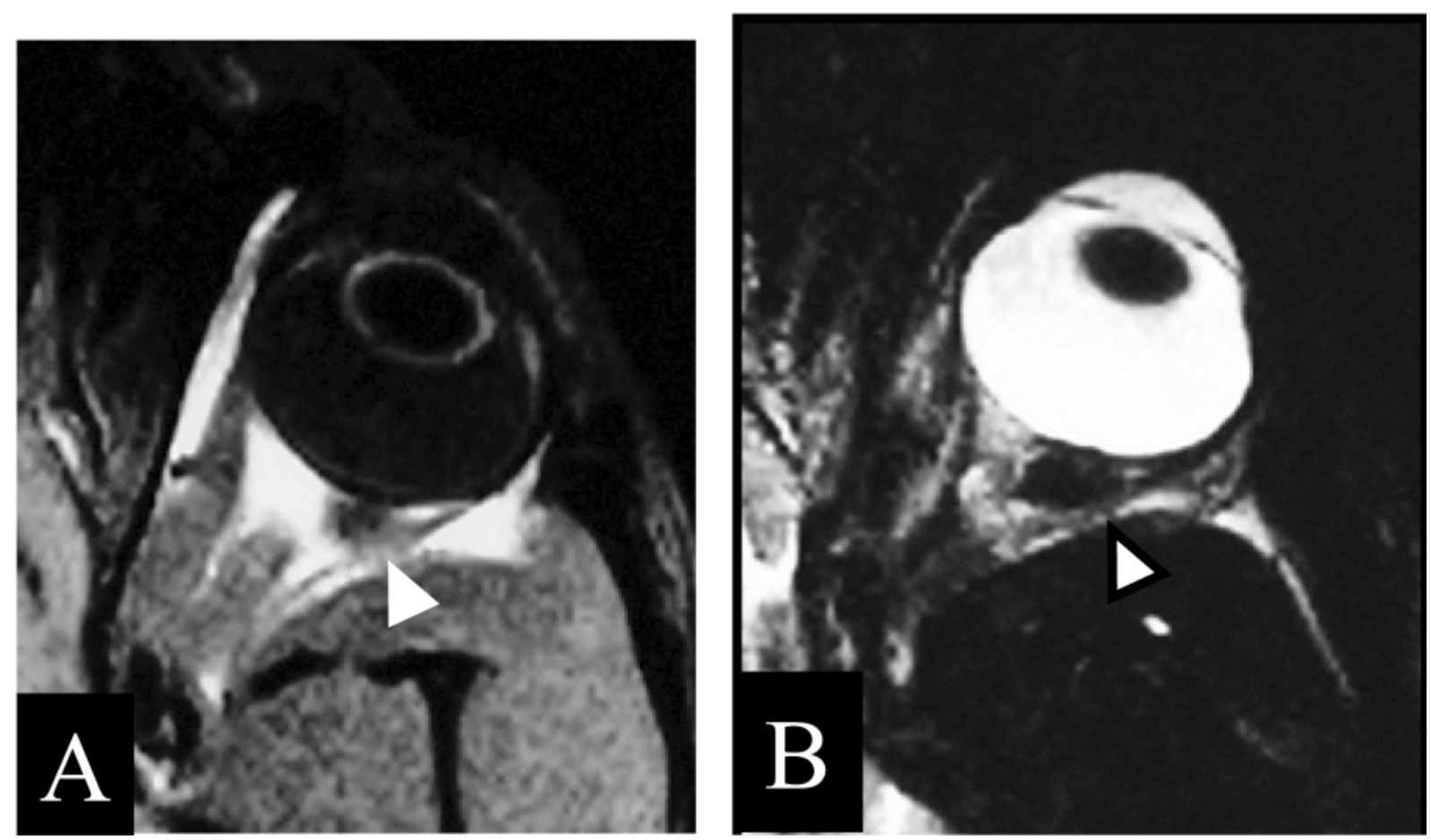

Fig. 2. A.B. Magnetic resonance images showing a mass (arrow head) posterior and over the optic disc of the left globe. The mass was hypointense with isointense margin on T1-weighted images (A) and hypointesne on T2-weighted images (B).

unit (MRP-7000 AD/AIRIS, Hitachi Medical Co., Tokyo, Japan). MR images revealed a mass overlaying the optic disc area, which proliferated toward the postorbital space. On T1-weighted images, the mass was isointense in its margin and hypointense in its central area (Fig. 2A). On T2weighted images, the mass was hypointense (Fig. 2B). Enhanced T1-weighted images after the intravenous administration of gadoliniumDTPA (Magnebisto ${ }^{\circledR}$ Nihon Shering, Inc. Osaka. $0.3 \mathrm{ml} / \mathrm{kg}$ ) revealed the mass with a slight enhancement in intensity.

A tentative diagnosis of intraocular neoplasia, with being strongly suspected to be melanoma, was made based on these examinations. Then the dog underwent orbital exenteration. The dog was recovered from surgery without any events and discharged in 3 days.

The tumor mass was solid, black in color and $7 \mathrm{~mm}$ in diameter, and located around the optic disk and infiltrated toward the postorbital area. Histopathology revealed diffuse proliferation of melanin-pigmented neoplastic cells in the choroid around the optic nerve, optic nerve itself and the surrounding fat and connective tissue, and extended to the postorbital space along the optic nerve (Fig. 3A). The tumor cells were also found in the vessels of the area. Bleached sections revealed that the tumor cells were relatively large and oval to multangular in shape and arranged in sheet. The nucleus of the tumor cells was small, clear and oval and mitotic figures were rare (Fig. 3B). Histopathologically the tumor was diagnosed as choroidal malignant melanoma with invasion into surrounding tissues including the optic nerve.

Three months after the surgery, MRI was undertaken, when the left orbital area was shown to be isointense to hyperintense on both T1- and T2-weighted images. On enhanced T1-weighted images, there was the enhanced area, however cytology by aspiration of the enhanced area revealed no neoplastic cells.

On examinations by 23 months of post operation, including thoracic radiographs, blood examinations and MR images, there were no signs of recurrence and metastasis and the dog was clinically healthy.

Choroidal melanoma is a relatively rare neoplasia in dogs, and most of cases previously reported were discovered casually in ophthalmoscopy for other purposes $[1,7]$. In the present case, the owner noticed vision loss of an affected eye, perhaps due to the location of the neoplasia. Most of canine ocular melanomas are benign, and in some of the cases with choroidal melanomas, no clinical signs appeared for several years without any treatments [7, 10]. In addition, as there are no avaiable data on whether enucleation increases or decreases the risk of metastasis [11], it is still controversial to do enucleation for a dog with normotensive and noninflammed globes containing melanoma. In the present case, repeated ophthalmoscopy revealed the enlarged black-pigmented area in two weeks and the precise imaging by ultrasonogram and MRI revealed the protruded mass in the postorbital space. Based on these findings, surgery of enucleation was undertaken.

MR images of the present case did not showed the char- 

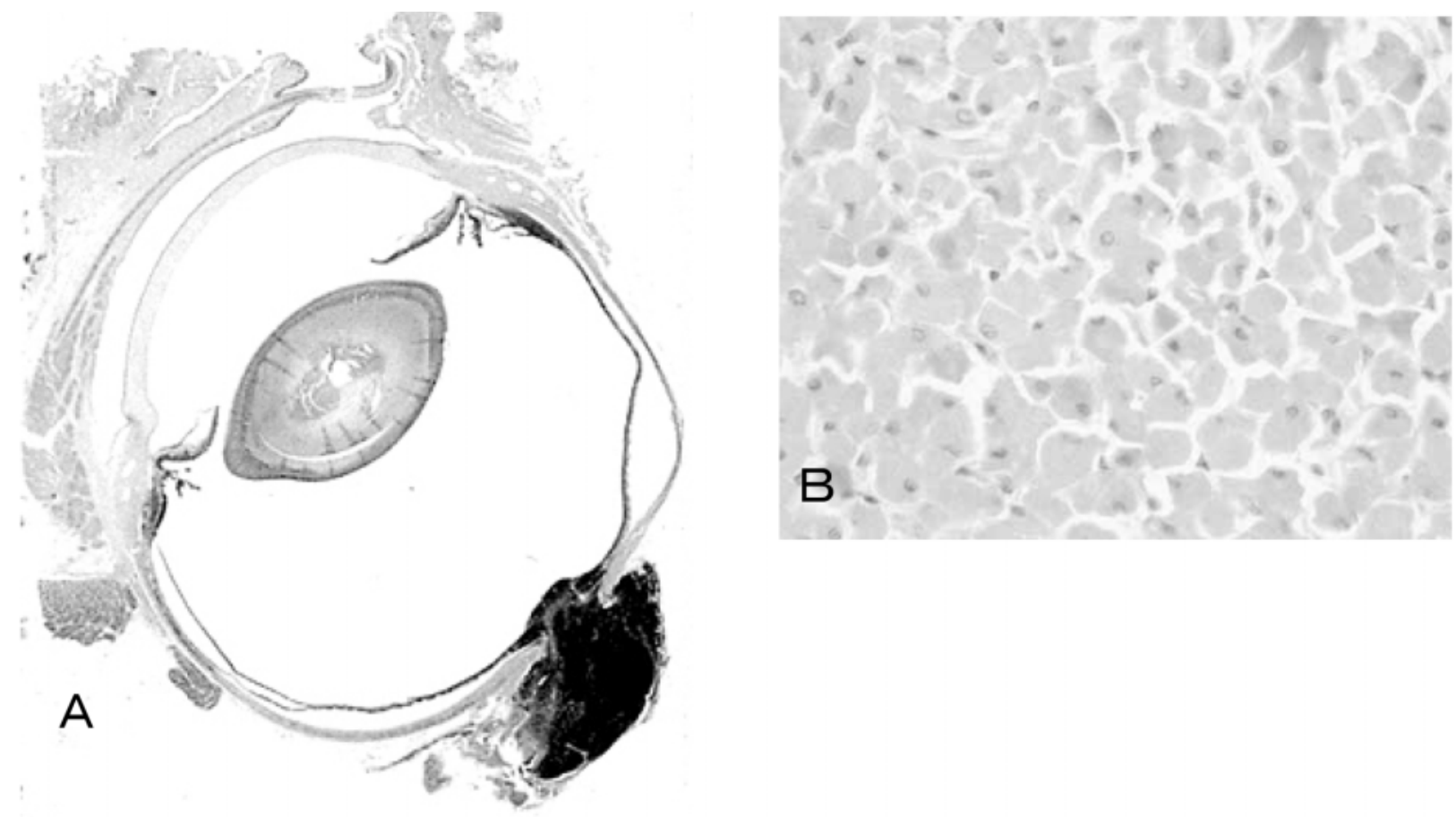

Fig. 3. A.B. Histological finding of the eye with a heavily pigmented melanoma. A: The tumor cells infiltrated into the posterior portion along the optic nerve (H. \& E.). B: Photomicrograph of a bleached section of the tumor. Note the relatively large and oval to multangular cells in shape with small, clear and oval nuclei (Bleached, H. \& E. $\times 200$ ).

acteristic image of the melanoma. MR images of melanoma masses are characterized by high signal intensity on T1weighted images and low signal intensity on T2-weighted images due to the presence of melanin $[5,8]$. In the present case, signal intensity of the lesion on T2-weighted images was low probably because of a large amount of pigments within the tumor cells. The signal intensity on T1-weighted images was not high. This may be due to a large amount of fat tissues existing in the orbit, which may affect the signal intensity of the lesion. It has been reported that the fat suppression technique of MR image is valuable when confirming the diagnosis of the extraocular melanoma $[6,8]$. This technique might be helpful to exclude possible artifacts in this case.

Postoperative enhanced T2-weighted images obtained 92 days after surgery showed enhancement at the left orbital area, and a recurrence was suspected, however cytology and the follow-up MR images obtained at 23 months postoperatively revealed no evidence of recurrence. The enhanced area might be an inflammatory change after surgery. However, the tumor cells were histologically found in the vessels of the tissue at the margin of the tumor when enucleated. In addition, delayed metastasis at 21 months after surgery was reported [4] in a canine case with choroidal melanoma, therefore further monitoring of recurrence should be conducted.

\section{REFERENCES}

1. Dubielzig, R.R. 1990. Vet. Clin. North Am. Small Anim. Pract. 20: $837-848$.

2. Dubielzig, R.R., Aguirre, G.D., Gross, S.L. and Diters, R.W. 1985. Vet. Pathol. 22: 582-585.

3. Giuliano, E.A., Chappell, R., Fischer, B. and Dubielzig, R.R. 1999. Vet. Ophthalmol. 2: 185-190.

4. Hyman, J. A., Koch, S.A. and Wilcock, B.P. 2002. Vet. Ophthalmol. 5: 113-117.

5. Kato, K., Nishimura, R., Sasaki, N., Matsunaga, S., Mochizuki M., Nakayama, H. and Ogawa, H. 2005. J. Vet. Med. Sci. 67: $179-182$.

6. Michael, S. and Vaphiades, D.O. 2001. Surv. Ophthalmol. 46: $56-58$.

7. O'Toole, D. and Murphy, J. 1983. J. Small Anim. Pract. 24: 561-567.

8. Recsan, Z., Karlinger, K., Fodor, M., Zalatnai, A., Papp, M. and Salacz, G. 2002. Clin. Radiol. 57: 371-376.

9. Schoster, J.V., Dubielzig, R.R. and Sullivan, L. 1993. J. Am. Vet. Med. Assoc. 203: 89-91.

10. Weisse, I., Frese, K. and Meyer, D. 1985. Vet. Pathol. 22: 586591.

11. Wilcocl, B.P. and Peiffer, R.L. 1986. Vet. Pathol. 23: 418-424. 